\title{
ADVANCED LYPOSARCOMA MYXOIDES OF THE EXTREMITY
}

\author{
Milan Petrović ${ }^{1}$, Ljiljana Jeremić ${ }^{1}$, Milan Radojković ${ }^{1}$, Ivica Pejčić ${ }^{2}$, \\ Ivona Djordjević ${ }^{3}$, Milica Marković ${ }^{4}$, Jelena Stojanović ${ }^{4}$ \\ ${ }^{1}$ General Surgery Clinic, Clinical Center Niš; Faculty of Medicine, University of Niš, Serbia \\ ${ }^{2}$ Oncology Clinic, Clinical Center Niš; Faculty of Medicine, University of Niš, Serbia \\ ${ }^{3}$ Pediatric Surgery Clinic, Clinical Center Niš, Faculty of Medicine, University of Niš, Serbia \\ ${ }^{4}$ Center for Anestesiology and Reanimation, Clinical Center Niš; Faculty of Medicine, University of Niš, Serbia
}

\begin{abstract}
Sarcomas are soft tissue tumors arising from primitive mesenchyme. Small incidence (4-5/100 000 in Europe) is the reason their pathogenesis is relatively unknown. Patient (38) complained of a growth on the upper part of right thigh, the size of a child's head. A magnetic resonance imaging (MRI) scan was done and tumefaction was described: oval shape, $13.5 \times 7.5 \times 11 \mathrm{~cm}$ in diameter, without infiltration of surrounding tissue. The tumor was surgically removed and was pathohistologically verified as low grade liposarcoma myxoides. After initial presentation the patient presented a series of recurrences and metastases in the abdominal wall, extremities and liver. Soft tissue metastasis from the lower extremities to the liver occur in $0.5 \%$ of cases and they are usually small and multiple, as in the presented patient. The European Sarcoma Medical Oncologist's guide recommends that every extremity tumor larger than 5cm and suspicious of malignancy should be evaluated using biopsy and imaging methods. A biopsy should be done before excision under ultrasound or computerized tomography $(C T)$ guidance with the goal of planning the best therapy protocol and prevention of a generalized disease with metastases. The recommended imaging method is an MRI scan, although sarcomas can have a benign presentation. Standard therapy includes surgical resection with local radiotherapy. Liposarcoma myxoides, a rare soft tissue tumor, demands biopsy and complete surgical removal with detailed and continuous postoperative imaging follow-up and oncological therapy. The therapeutic goal is to increase survival and preserve extremity function.
\end{abstract}

Key words: Sarcoma, Liposarcoma.

\section{Introduction}

Adult soft tissue sarcomas are malignant tumors which develop from primitive mesenchyme. Their incidence is small (around 4-5/100000 cases per year in Europe) [1] which is the main reason their pathogenesis and metastasizing pattern is relatively unknown, and that is also the reason why we have few large randomized prospective studies on this topic. Therefore, scientific papers in the form of case presentations are a valuable source of information in the ongoing research of this disease.

\section{Case Presentation}

The patient (38 years old) complained to his general practice doctor in July 2013 of a large growth the size of a child's head on the inner side of his right thigh. After a physical exam and laboratory analysis of blood and urine, a magnetic resonance imaging (MRI) scan of the lower extremities was done, which described a tumefaction beneath the pubic bone and between the obtura-

Correspondence to: Ivona Djordjević, Ph.D.

Pediatric Surgery Clinic, Clinical Center Niš, Serbia

48 Dr. Zoran Djindjić Blvd., Niš 18000, Serbia

Phone: +38163812 2532

E-mail: ivonadj74@gmail.com

Received May $6^{\text {th }}, 2017$, Accepted October $16^{\text {th }}, 2017$ tor muscles: clear margins, oval shape, and $13.5 \times 7.5 \mathrm{x}$ $11 \mathrm{~cm}$, without infiltration of surrounding tissue, benign in appearance. After one month the tumor was surgically removed. The tumor was pathohistologically verified as a primary mesenchyme tumor type low grade myxoid liposarcoma. Tumor immunophenotipisation: CD34-, p53-, Cyclin D1+, rear individual cells $<1 \%$, $\mathrm{Ki}-67+$ diffuse heterogeneous weak to intensive immunopositivity in around $15 \%$ of tumor cells. He was referred to a multidisciplinary team in September 2013 which indicated a follow up every two months with regular MRI scans. After ten months, a recurrence was diagnosed, when a reoperation was done as well as regional lymph node dissection. After that, the multidisciplinary team indicated postoperative local radiotherapy (RT) which was not conducted due to technical reasons. In the meantime, the patient was diagnosed with a tumor in the abdominal wall. He was operated on for the third time in July 2014 where the abdominal wall tumor was removed and the pathohistological finding was the same as before (low grade liposarcoma myxoides). The multidisciplinary team requested a postoperative computerized tomography (CT) scan of the thorax and abdomen. A suspicious lesion was then noticed in the liver as well as a group of enlarged lymph nodes in the mediastinum. MRI of the right thigh did not show any sign of recurrence. A positron emission tomography (PET) scan was 
done which showed a nodular tumefaction in the neck on the left posterior side in the subcutaneous tissue $12 \mathrm{~mm}$ in diameter as well as a circular defect in the medial part of hind knee area in the subcutaneous tissue. The multidisciplinary team indicated systemic chemotherapy in three cycles using monotherapy with Doxorubicin. Because of a new tumefaction in the left elbow and a recurrence in the primary surgical site, the chemotherapy was continued for three more cycles, but was aborted due to cardiotoxity. The above-mentioned recurrence was surgically removed in September 2015. RT was again not applied. This was followed by recurrence in January 2016. CT scan of the thorax, abdomen and pelvis was done confirming lesion in the second segment of liver $(5.7 \mathrm{~cm})$ (Fig. 1) as well as in the right inguinal area.

Five months after that, ultrasound of the abdomen showed enlargement of the solitary soft tissue lesion in the left lobe of the liver $(9.8 \times 9.2 \mathrm{~cm})$. The patient was referred to our clinic for further treatment of the liver lesion in July 2016. After adequate preoperative preparation, „J" laparotomy was performed (with continuous ischemia using the Pringle maneuver), finding a large solid tumefaction in the left lobe of the liver $10 \times 8 \mathrm{~cm}$ in size. The tumor was verified as a myxoid liposarcoma metastasis. In the follow up course the patient was diagnosed with multiple liver and lung metastases which were verified with CT scans. Table 1 is showing all the therapeutic and diagnostic procedures performed in the patient in chronological order.

\section{Discussion}

Liposarcoma is the second most common soft tissue sarcoma. The exact cause of their occurence is unknown. Patients usualy first notice a growth on the extremity after an injury. However it is certain that liposarcomas do not occur because of trauma. They most often occur between 30 and 50 years of age with a mild male predominance. They can occur anywhere in

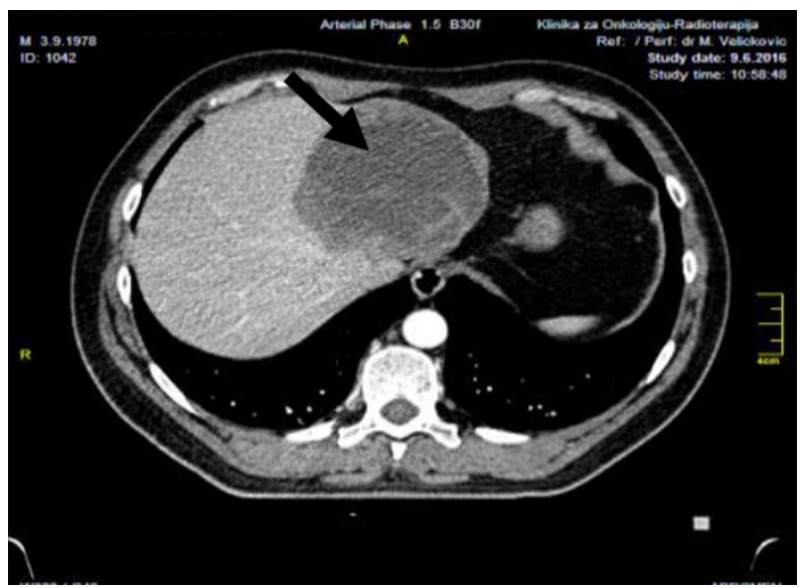

Fig. 1 Transverse abdominal CT scan. Large metastasis is visible in second segment (black arrow)
Table 1 Overview of the diagnostic and therapy procedures.

\begin{tabular}{ll}
\hline Disease events & $\begin{array}{l}\text { Time } \\
\text { period }\end{array}$ \\
\hline $\begin{array}{l}\text { Operation- excision of the growth in the } \\
\text { right thigh (PH: low grade myxoid }\end{array}$ & $\begin{array}{l}\text { August } \\
\text { liposarcoma) }\end{array}$ \\
$\begin{array}{l}\text { Operation- excision of the recurrent growth } \\
\text { on the right thigh with dissection of the }\end{array}$ & May 2014 \\
regional lymph nodes (PH: low grade \\
myxoid liposarcoma)
\end{tabular}

the body where fat tissue is present, most commonly in the deep fat tissue of the extremity (59\%), abdomen and thorax $(19 \%)$, retroperitoneum $(15 \%)$, head and neck $(9 \%)$ [2]. The greatest survival rate is with liposarcoma of the extremities and the least with retroperitoneal sarcomas. The local recurrence rate is arround 78\% [3].

According to the histology type, liposarcomas can be classified as: dedifferentiated, myxoid and pleomorphic [4]. Myxoid liposarcoma is the most common subtype and it is more frequent in younger people. It is different from other liposarcomas in its unusual pattern of metastasis in tissues such as: bones (most common), retro-peritoneum, extremities and the axillary region, while other localities are affected only in advanced stages of the disease [5,6].

All other liposarcomas usually present with metastases primarily in the lungs. Soft tissue sarcoma metastases of the lower extremities occur in the liver in around $0.5 \%$ of cases [7] and usually present as small multiple metastases and rarely as big solitary tumors such as the one in the patient described in this presentation. 
According to the European society of medical oncologists (ESMO), every patient with a tumor suspicious for soft tissue sarcoma of the extremity should be referred to a specialized diagnostic center for a threeway evaluation: biopsy, physical exam and imaging [8]. The recommended imaging method for evaluation is MRI, which was used for diagnosing the presented patient as well. However, initially the tumefaction was described as a benign tumor lesion with clear margins and no infiltration of surrounding tissue. As shown by Francois et al. myxoid liposarcomas, although malignant tumors, can deceive with their benign appearance [9]. For this reason, any growth which is larger than $5 \mathrm{~cm}$ and deeply seated in the tissue should be suspicious for malignancy. An important diagnostic tool which is also used for planning further therapy is a tumor biopsy which should be done before tumor excision. The first biopsy is performed under ultrasound or CT guidance and in case of failure, a surgical biopsy is needed. The histological classification should be made according to the World Health Organization classification from 2013. The grade of the malignancy should always be determined if possible, because it has prognostic value. The grade is determined based on degree of necrosis, differentiation of the tumor and mitosis count [10].

As far as other diagnostic modalities are concerned, the ESMO guide recommends CT of the thorax, for patients who were diagnosed with soft tissue sarcoma by biopsy, in order to rule out pulmonary metastases before definitive treatment. CT of the abdomen and pelvis do not have to be done routinely, but they are recommended in certain histological types, including myxoid liposarcomas. Early diagnostics could lead to earlier discovery of metastatic disease, and an earlier introduction of systemic chemotherapy.

Wide excision with negative margins in combination with postoperative radiotherapy is the standard in treating tumors of the trunk and extremity because that provides a high degree of local control with preservation of extremity function. The margin of surgical excision depends on multiple factors: histological subtype of the tumor, application of preoperative chemo or/and radiotherapy and presence of anatomical barriers (fascia, periost etc.). In some cases amputation of the extremity should be considered. Although radiation therapy is reserved mostly for patients with high grade tumors, it can be justified in low grade tumors which are deeply seated in the tissue and larger than $5 \mathrm{~cm}$. This decision should be made by a multidisciplinary team taking in consideration the anatomical localization of the tumor, as well as the risk/benefit ratio in radiating patients with a low grade tumor. Local radiation therapy reduces the incidence of local recurrence which is often related to metastatic disease. The presented patient did not receive any local radiation therapy due to technical reasons, which may have led to multiple recurrences and the generalized metastatic disease. However, since this is a case presentation and this is only one case, it is difficult to draw conclusions and determine correlation between the absence of radiotherapy and advancement of the disease.
Neoadjuvant radiotherapy is not recommended as a routine method, but it can be used in radiosensitive subtypes such as myxoid liposarcomas in order to shrink the tumor preoperatively. The main drawbacks of this method are postoperative surgical complications which occur at the site of tumor excision.

The role of adjuvant chemotherapy has not been confirmed, therefore it is also not recommended as a routine method. It can however be used in patients whose tumor is near an anatomical structure which could be damaged using radiotherapy or in certain chemo-sensitive tumors.

Advanced disease with extrapulmonary metastases is best treated with systemic chemotherapy. In certain patients, metastases can be treated surgically, depending on the localization of the tumor and progression of the disease. Standard chemotherapy used today is Doxorubicin as a monotherapy, since it has not been formally proven that polytherapy is superior to monotherapy. On the other hand, some studies are in favor of polytherapy in certain chemo-sensitive types of tumors [11]. The most dangerous side effect of Doxorubicin is cardio toxicity, which was observed in the presented patient.

As far as follow up is concerned, the ESMO guide recommends that all patients with low grade tumors should be controlled every four to six months in the first three to five years and then once a year. Standard control should encompass a physical exam (and MRI scan if needed), as well as a chest $X$ ray to rule out pulmonary metastases.

Regarding prognosis in these patients, negative prognostic factors are: age ( $>45$ years) [12], size of the tumor $(>10 \mathrm{~cm})$ [13], percentage of differentiation of cells $(>5 \%)[14]$ and tumor necrosis [12]. All of these factors were present in the presented patient except his age.

\section{Conclusion}

Liposarcomas rarely metastasize from the extremity to the liver. When they do, it is usually in the form of multiple metastases, and rarely as large solitary tumefactions, which is a sign of an advanced stage of the disease. According to the ESMO guide, if there is suspicion of a soft tissue sarcoma, it is necessary to do an ultrasound or CT guided biopsy before excision in order to plan a therapeutic protocol based on the pathohistological diagnosis. In order to prevent recurrence, it is necessary to consider local radiotherapy after surgical excision in tumors that are primary, deeply seated and larger than $5 \mathrm{~cm}$ even though they are low grade. Also, it is recommended to do a CT or a chest X-ray of the thorax and in subtypes such as liposarcoma myxoides a CT of the abdomen and pelvis as well, in order to rule out the presence of metastases. Systemic chemotherapy is the method of choice in treating advanced disease with extra-pulmonary metastases and in certain cases, surgical therapy can be performed. Follow up in low grade tumors should be done every six months in the first two to three years with a thorough physical exam and a chest X-ray. 


\section{References}

1. Stiller CA, Trama A, Serraino D, Rossi S, Navarro C, Chirlaque MD. Descriptive epidemiology of sarcomas in Europe: Report from the RARECARE project. Eur J Cancer 2013; 49(3):694-5.

2. Cormier JN, Pollock ER. Soft tissue sarcomas. CA Cancer J Clin 2004; 54:94-109.

3. Celik C, Karakousis CP, Moore R, Holyoke ED. Liposarcomas: Prognosis and Management. J Surg Oncol 1980; 14:245-9.

4. Fletcher CDM et al. WHO classification of tumours of soft tissue and bone. Lyon: IARC 2003:305-10.

5. Asano N, Susa M, Hosaka S, Nakayama R, Kobayashi E, Takeuchi $\mathrm{K}$ et al. Metastatic patterns of myxoid/round cell liposarcoma: a review of a 25-year experience. Sarcoma 2012.

6. Schwab JH, Boland P, Guo et al. Skeletal meastases in myxoid liposarcoma: an unusual pattern of distant spread. Ann Surg Oncol 2007; 14(4):1507-14.

7. Jaques D, Coit D, Casper E, Brennan M. Hepatic Metastases from Soft-Tissue Sarcoma. Ann Surg 1995; 4:392-7.
8. The ESMO / European Sarcoma Network Working Group. Soft tissue and visceral sarcomas: ESMO Clinical Practice Guidelines for diagnosis, treatment and follow-up. Ann Oncol 2014; 25(3): 102-12.

9. Loubignac F, Bourtoul C, Chapel F. Myxoid liposarcoma: a rare soft-tissue tumor with a misleading benign appearance. World $\mathrm{J}$ Surg Oncol 2009; 7:42.

10. Trojani M, Contesso G, Coindre JM, et al. Soft-tissue sarcomas of adults: study of pathological prognostic variables and definition of a histopathological grading system. Int J Cancer 1984; 33:37-42.

11. Antman K, Crowley J, Balcerzak SP, et al. An intergroup phase III randomized study of doxorubicin and dacarbazine with or without ifosfamide and mesna in advanced soft tissue and bone sarcomas. J Clin Oncol 1993; 11:1276-85.

12. Kilpatric SE, Doyon J, Choong PFM Sim FH, Nascimento AG. The clinicopathologic spectrum of myxoid and round cell liposarcoma: a study of 95 cases. Cancer 1996; 77(8): 1450-8.

13. Orson GG, Sim FH, Reiman HM, Taylor WF. Liposarcoma of the musculoskeletal system. Cancer 1987; 60(6):1362-70.

14. Reitan JB, Kaalhus O, Brennhovd IO. Prognostic factors in liposarcoma. Cancer 1985; 55(10):2482-90. 